stomatologie 2014 · 111:175-181 DOI 10.1007/s00715-014-0335-4

Online publiziert: 9. Juli 2014

(c) The Authors 2014

This article is published with open access at Springerlink.com

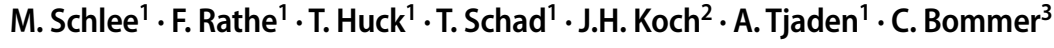 \\ 1 Praxis Dr. Markus Schlee, Forchheim, Deutschland \\ ${ }^{2}$ Freising, Deutschland \\ ${ }^{3}$ credentis ag, Windisch, Schweiz
}

\title{
Klinischer Effekt biomimetischer Mineralisation bei Approximalkaries
}

\section{Halbjahresergebnisse einer klinischen Studie}

\section{Einleitung}

\section{Epidemiologie der Karies}

In der westlichen Welt ist die Karieshäufigkeit in den letzten Jahrzehnten bei Anwendung klassischer Kriterien (DMF/ dmf) deutlich zurückgegangen. Dennoch ist sie nach wie vor ein ungelöstes Problem, was z. B. mit einem Wert von über $50 \%$ bei deutschen 12-Jährigen deutlich wird [21]. Hinzu kommt eine deutlich Polarisierung mit $61,1 \%$ der Karieserfahrung bei nur 10,2\% der Probanden [21]. Initiale, nichtkavitierte Läsionen sind zudem mit DMF/dmf-Kriterien nicht messbar. Daher könnte die tatsächliche Anzahl kariöser Läsionen im Vergleich zu früheren Jahrzehnten unverändert hoch sein $[6,7]$.

Neben okklusalen sind approximale Flächen bleibender Zähne am häufigsten betroffen. So traten in einer schwedischen Gruppe prophylaktisch intensiv betreuter 12- bis 15-Jähriger flächenbezogen 4,3\% neue Schmelzläsionen pro Jahr auf [17]. Die jährliche Rate der Läsionen, die sich von der Schmelz-Dentin-Grenze in die äußere Dentinhälfte ausgebreitet hatten, lag in derselben Altersgruppe bei 32,5\%. Sobald ein kariöser Prozess das Dentin erreicht, muss mit einer schnelleren Ausbreitung des kariösen Defekts in Richtung Pulpa gerechnet werden [4]. Die meist aus Kunststoffen oder Keramik bestehenden Restaurationen müssen laufend repariert oder erneuert werden und die Prognose wird mit zunehmender Füllungsgröße schlechter [27]. Die weltweiten Kosten einer mechanisch orientierten Kariesversorgung sind erheblich und die gesundheitlichen Folgen betreffen überwiegend sozial benachteiligte Bevölkerungsgruppen [23].

\section{Remineralisation und Remineralisationsförderung}

Um die Kariesinzidenz zu senken, wird der Einsatz von Fluoriden als Goldstandard seit Jahrzehnten intensiv erforscht $[14,24]$. In einer systematischen Literaturübersicht wurde für systemisch und lokal wirkende Fluoride ein primärer kariespräventiver Effekt zwischen 22\% und 29\% festgestellt [9]. Die lokale Wirkung von Fluoriden kommt vor allem bei aktiven Initialläsionen zum Tragen [22]. Hier ist bereits eine Demineralisierung vorhanden und die Fluoride wirken entsprechend sekundär präventiv [10]. Da kariöse Demineralisation ein dynamischer Prozess ist, lassen sich primäre und sekundäre Wirkungen nicht eindeutig trennen. Die therapeutisch wirksame Fluoridkonzentration ist, auch bei hochkonzentrierten Produkten für die professionelle Anwendung, maximal für $24 \mathrm{~h}$ gegeben [5]. Bei erhöhtem Risiko und Verschiebung der oralen Balance in Richtung Karies werden daher zusätzliche Maßnahmen wie intraorale Träger mit kontrollierter Fluoridabgabe oder antimikrobielle Substanzen empfohlen [7, 8].

\section{》) Die Fluoridkonzentra- tion ist maximal für $24 \mathrm{~h}$ therapeutisch wirksam}

Mithilfe in letzter Zeit eingeführter remineralisierender Produkte soll die Kariesbalance ebenfalls günstig beeinflusst und damit verhindert werden, dass kariöse Läsionen entstehen oder fortschreiten. So

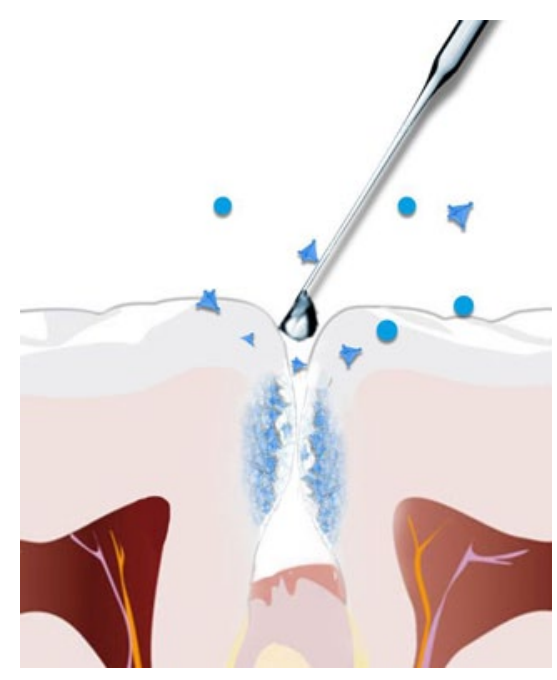

Abb. $1 \Delta$ Wirkungsprinzip der biomimetischen Mineralisation. Nach Auftragen sich selbst organisierender Peptide in wässriger Lösung entsteht nach Diffusion im Läsionskörper eine organische Matrix, die durch ihre hohe Affinität zu Kalzium- und Phosphationen (dargestellt als blaue Sphären respektive blaue Tetraeder) die Neubildung von naturanaloger Zahnhartsubstanz ermöglicht und so die kariöse Läsion mineralisiert. (Mit freundl. Genehmigung von credentis) 


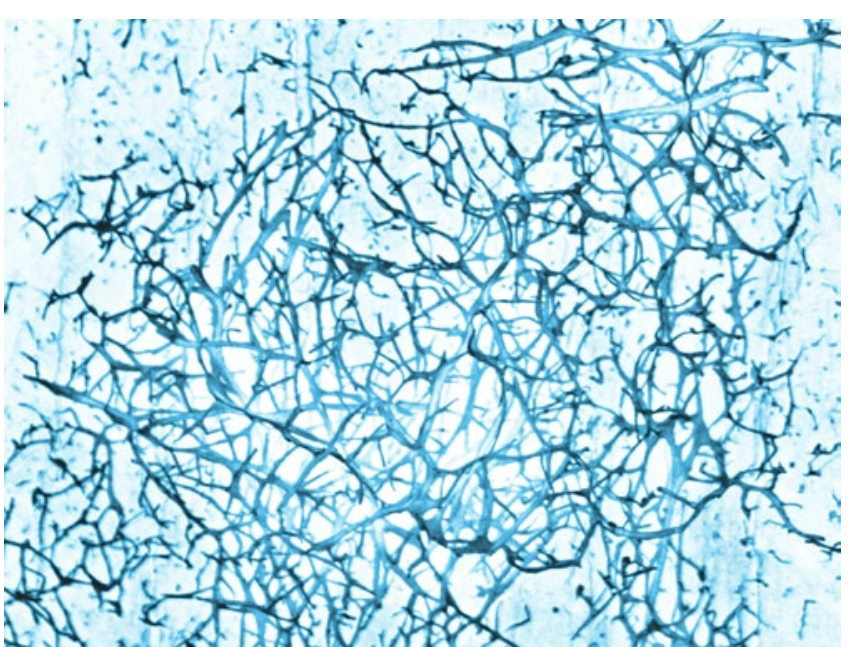

Abb. $2<$ Die nanofibröse Matrix ist durch Poren und Tunnel charakterisiert und aufgrund ihrer chemischen Zusammensetzung resistent gegen bakterielle Enzyme. (Rasterelektronenmikroskop, Vergr. 120.000:1; mit freundl. Genehmigung von A. Aggeli und S. Maude, Universität Leeds)

wurde für die Wirkstoffkombination Kaseinphosphopeptid/amorphes Kalziumphosphat (CPP/ACP, Recaldent ${ }^{\mathrm{Tm}}$, GC Europe, Leuven, Belgien) ein kariesprotektiver Effekt bei Initialläsionen gezeigt [3, 28]. Da Kalzium und Phosphat bei gesunden Patienten in gesättigter Konzentration, bei vermindertem Speichelfluss aber nur begrenzt verfügbar sind, könnte diese Wirkung vor allem bei Patienten mit Hyposalivation zum Tragen kommen [7].

Der remineralisationsfördernde Effekt von Fluoriden und die Wirkung remineralisierender Substanzen beschränkt sich i.d.R. auf die äußere hypermineralisierte Schicht, unter der der kariös bedingte $\mathrm{Ab}$ bau von Mineralen stattfindet [1]. Wünschenswert ist jedoch nach aktuellen Einschätzungen, dass auch tiefere Schichten durch Einlagerung von Mineralen stabilisiert werden $[3,25]$. Idealerweise sollte dadurch nicht nur die Progression der Läsionen gestoppt, sondern eine in der Tiefe messbare Rückentwicklung erreicht werden.

\section{Versiegelung und Infiltration}

Mithilfe konventioneller Schmelz-Dentin-Adhäsive lassen sich initialkariöse Approximalflächen nach forcierter Separation mit Keilen versiegeln [15]. Diese Methode verhindert das Fortschreiten der Läsion über einen Zeitraum von 3 Jahren signifikant wirksamer, als wenn keine Behandlung durchgeführt wird [16]. Die Auswertung der Ergebnisse erfolgte anhand direkter Bewertung von Bissflügelaufnahmen und Subtraktionsradiographie.
Alternativ steht die Infiltration mit einem speziellen dünnflüssigen Kunststoff zur Verfügung, der sowohl approximal als auch auf Glattflächen anwendbar ist (Icon, DMG, Hamburg) [18]. Nach Vorbehandlung der Schmelzoberfläche mit Salzsäure wird die aufgelockerte Schmelzstruktur von dem Kunststoff durchdrungen. In einer kontrollierten Studie unter universitären Bedingungen konnte gezeigt werden, dass radiologisch nach 3 Jahren nur eine von 26 Läsionen fortgeschritten war [19]. Die hypermineralisierte Schmelzoberfläche wird durch die Salzsäure bis zu einer Tiefe von ca. $40 \mu \mathrm{m}$ und damit in den meisten Fällen annähernd vollständig abgetragen [20]. Zudem dringt der Kunststoff im Gegensatz zu Fissurenversiegelungen in den Schmelz ein. Daher ist die Kunststoffinfiltration als minimal- oder mikro-invasive Behandlung einzustufen.

\section{Biomimetische Mineralisation}

Seit dem Jahr 2012 steht mit der biomimetischen Mineralisation eine neue Methode für die Behandlung von Initialkaries zur Verfügung, die ohne Kunststoffe auskommt. Mithilfe eines sich selbst organisierenden, organischen Moleküls („,selfassembling peptide“, SAP) wird eine Neubildung von Schmelzkristallen im Defekt gesteuert (Curodont Repair, credentis, Windisch, Schweiz) [12]. Das im Produkt enthaltene Peptid (P11-4) wird in wässriger Lösung auf den mit 35\%iger Phosphorsäure (Ätzgel, Orbis Handel GmbH, Münster, Deutschland) geätzten, oberflächlich intakten Schmelz aufgetragen und dringt in den Läsionskörper ein
(- Abb. 1). Dort baut es in wenigen Minuten sich selbst organisierend eine biologische Matrix auf (• Abb. 2). Dieses dreidimensionale Netzwerk besitzt eine hohe Affinität zu Kalzium- und Phosphationen. Im Verlauf der folgenden Wochen und Monate ermöglicht es die Mineralisation der frühen Karies bis in die Tiefe durch Neubildung von naturanaloger Zahnhartsubstanz aus Kalzium- und Phosphationen aus dem Speichel, was an einem erhöhten Mineralgehalt ablesbar ist [12].

Nach In-vitro-Daten findet damit erstmals ein Remineralisation in der Tiefe des Defekts statt [11]. Die klinische Wirksamkeit der Methode wurde bereits in einer Pilotstudie an der Universität Leeds gezeigt [2]. Die Größe von White-Spot-Läsionen konnte darin über einen Zeitraum von 30 Tagen signifikant reduziert werden und war für 6 Monate stabil. Gleichzeitig wurden die Läsionen im Vergleich zum Ausgangsbefund als signifikant verbessert bewertet (verblindetes Verfahren, keine Kontrollgruppe). Die vorliegende Publikation beschreibt - als Zwischenbericht einer auf 1 Jahr angelegten Studie die Wirkung der biomimetischen Mineralisation bei Anwendung in approximalen Initialläsionen unter Praxisbedingungen nach 6 Monaten.

\section{Material und Methode}

\section{Ethikvotum, Patientengut und Endpunkt}

Die prospektive, unkontrollierte Studie wurde gemäß ISO 14155:2011 und nach den Vorgaben der Deklaration von Helsinki durchgeführt. Sie wurde durch die Freiburger Ethik-Kommission International genehmigt (FEKI, Code 012/1035).

Die Studiengruppe, rekrutiert als konsekutive Fallserie, umfasste zu Beginn 25 Patienten der Praxis Dr. Markus Schlee, mit je einer oder mehreren unbehandelten approximalen Initialläsionen. Bei der Zwischenauswertung nach 6 Monaten standen 21 Patienten (durchschnittliches Alter 35 Jahre; 9 männlich, 12 weiblich) mit insgesamt 26 Läsionen zur Verfügung.

Als primärer Endpunkt der Untersuchung wurde die radiologische Veränderung gegenüber dem Ausgangsbefund 
nach Ablauf von 6 und 12 Monaten festgelegt (definitive Auswertung). Als sekundärer Endpunkt diente für die vorliegende Zwischenauswertung die Ausdehnung der Läsion in Schmelz und Dentin [15].

\section{Ausgangsbefunde und klinisches Vorgehen}

Der durchschnittliche DMF-T der Probanden betrug 9,0 4 4,9. Die 26 Läsionen wurden zu Beginn (Zeitpunkt D0) nach radiologischer Flächenausdehnung den Kategorien äußere (E1) oder innere (E2) Schmelzhälfte oder äußeres Dentindrittel (D1) zugeordnet (E1: 5 Läsionen, E2: 20 Läsionen, D1: 1 Läsion, s. • Abb. 5b, [15]). Hierfür wurden mithilfe von individualisierten Röntgenfilmhaltern (Rinn, Dentsply DeTrey, Konstanz) standardisierte digitale Bissflügel- und intraorale Einzelfilmaufnahmen erstellt und ausgewertet.

Alle Läsionen wurden zu Beginn (Zeitpunkt D0) mit dem Inhalt von je 1 Ampulle des Testprodukts in $50 \mu \mathrm{l}$ wässriger Lösung (Curodont Repair) behandelt (- Abb.3). Hierfür wurde der Defektbereich zunächst mit Kofferdam oder Speichelabsorbern (Dry Tips, Mölnlycke Health Care, Göteborg, Schweden) trockengelegt (Assistenz) und mit Finierstreifen (Epitex Extra Fine, GC Europe, Leuven, Belgien) vorgereinigt. Nach Separation des Interdentalraums mit einem Holzkeil wurden organische Ablagerungen mit 2\%igem Natriumhypochlorit $(\mathrm{NaOCl})$ abgewaschen ( $\bullet$ Abb. 3a) und die Flächen für $20 \mathrm{~s}$ mit 35\%iger Phosphorsäure geätzt ( $\bullet$ Abb. 3b). Die Säure wurde schließlich gründlich mit Wasserspray abgespült ( $\bullet$ Abb. 3c) und mit komprimierter Luft getrocknet. Die Testsubstanz wurde dann je 2-mal mit der dafür vorgesehenen Kanüle interdental appliziert (• Abb. 3d). Zwischen der ersten und zweiten Applikation wurde eine Einwirkzeit von jeweils 2,5 min eingehalten, nach der zweiten von $5 \mathrm{~min}$.

Nach Ablauf von 6 Monaten (D180) wurden erneut Röntgenaufnahmen mithilfe der vorhandenen individualisierten Filmhalter erstellt.

stomatologie 2014 · 111:175-181 DOI 10.1007/s00715-014-0335-4

(C) The Authors 2014. This article is published with open access at Springerlink.com

M. Schlee - F. Rathe - T. Huck - T. Schad - J.H. Koch · A. Tjaden - C. Bommer Klinischer Effekt biomimetischer Mineralisation bei Approximalkaries. Halbjahresergebnisse einer klinischen Studie

\section{Zusammenfassung}

Hintergrund. Karies hat bis heute eine hohe Prävalenz, auch in westlichen Ländern mit präventiven zahnmedizinischen Angeboten. Bei mechanischer Therapie werden die behandelten Zähne geschwächt. Dies führt über die Jahre oder Jahrzehnte häufig zum Verlust mit erheblichen Folgekosten. In den letzten Jahren eingeführte remineralisierende Substanzen sollen die etablierten remineralisationsfördernden Fluoride ergänzen. Daneben stehen die nicht oder minimal-invasiven Konzepte approximale Versiegelung und Kunststoffinfiltration zur Verfügung. Methode. In diesem Beitrag wird mit der biomimetischen Mineralisation eine neue Methode vorgestellt, die auf sich selbst organisierenden Peptiden (SAP) beruht. Die SAP werden in wässriger Lösung auf die gereinig- te und geätzte Schmelzoberfläche aufgetragen und dringen in den Läsionskörper ein. Dort bauen sie eine organische Matrix auf, die sekundär mit Kalzium- und Phosphationen aus dem Speichel mineralisiert wird. Ergebnis. Die Zwischenauswertung einer klinischen Studie bei Approximalläsionen zeigt, dass mit der neuen Methode drei von vier Läsionen stabilisiert oder remineralisiert werden konnten. Damit könnte es erstmals gelingen, demineralisierten Schmelz bis zum angrenzenden Dentin naturanalog wieder herzustellen.

\section{Schlüsselwörter}

Remineralisation · Zahnschmelz .

Biomimetik · Karies · Peptide

\section{Clinical effect of biomimetic mineralization in approximal caries. Results of a clinical study after 6 months}

\section{Abstract}

Background. Caries still has a high prevalence even in western countries with preventive dental care. When treated mechanically carious teeth will be weakened, which in the long-term often results in tooth loss and substantial inherent costs. In recent years various remineralizing products have been introduced which are supposed to supplement fluorides which in turn promote remineralization. Moreover, specific invasive or minimally invasive options are available, such as proximal sealing and caries infiltration.

Methods. This article presents a new method of biomimetic mineralization based on self-assembling peptides (SAPs). Suspended in water the SAPs are applied onto the cleaned and etched enamel surface. From there the SAPs will diffuse into the lesion and trigger the formation of a bioactive scaffold, which in turn encourages natural repair through incorporation of calcium and phosphate ions from the saliva.

Results. According to interim results from a clinical study using biomimetic mineralization, three out of four proximal lesions were stabilized or remineralized. The new method may allow for the first time natural repair of demineralized enamel extending to the enamel-dentin junction.

\section{Keywords}

Tooth remineralization - Enamel . Biomimetics · Caries · Peptides

\section{Klinische Bewertung und statistische Auswertung}

Die Bilder wurden vor der Bewertung paarweise auf Kontrast und Helligkeit kalibriert (Software ImageJ; Version 1.47k, Wayne Rasband, NIH, USA). Abweichungen zwischen den Röntgenbildern vom Ausgangsbefund (D0) und nach 180 Tagen (D180), die eine Beurteilung behindern konnten, mussten ausgeglichen werden.
Die klinische Bewertung der Röntgenbilder, die vorher am Tag der Bewertung kalibriert wurden, erfolgte durch 3 Untersucher (FR, TS und TH). Die Bewerter waren hinsichtlich des Aufnahmezeitpunkts der Röntgenbilder (D0 und D180) verblindet. Der Gesamteindruck der radiologischen Veränderungen (primärer Endpunkt) und die Flächenveränderungen (sekundärer Endpunkt) wurden durch paarweisen, randomisierten Ver- 

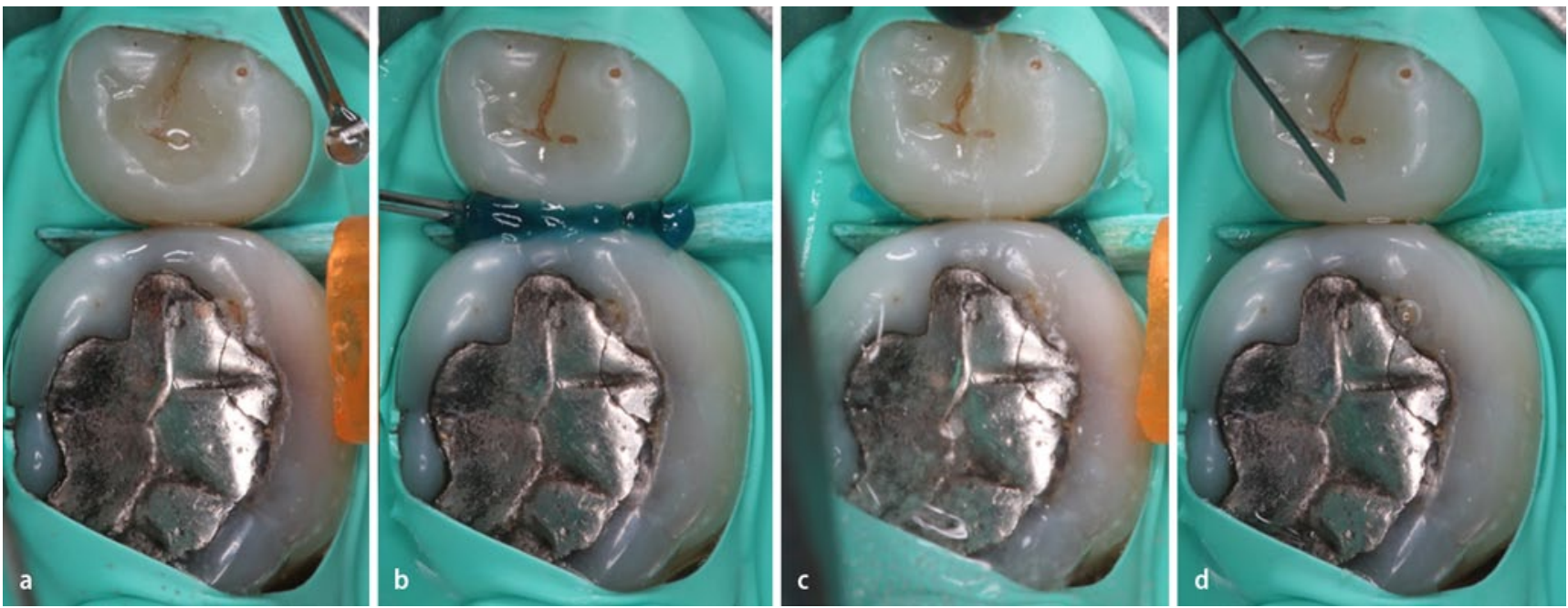

Abb. $3 \Delta$ Klinische Anwendung sich selbst organisierender Peptide an einer Approximalfläche mit Initialläsion. Der Defektbereich wird zunächst mit einem feinen Sandpapierstreifen und a anschließend mit Natriumhypochlorit ( $\mathrm{NaOCl}$ ) vorgereinigt. Nach Abspülen des $\mathrm{NaOCl}$ folgt b eine Konditionierung mit Phosphorsäure, $\mathbf{c}$ Abspülen, dann Trocknen und d die Applikation der wässrigen Peptidlösung. (Mit freundl. Genehmigung Praxis Dr. M. Schlee)

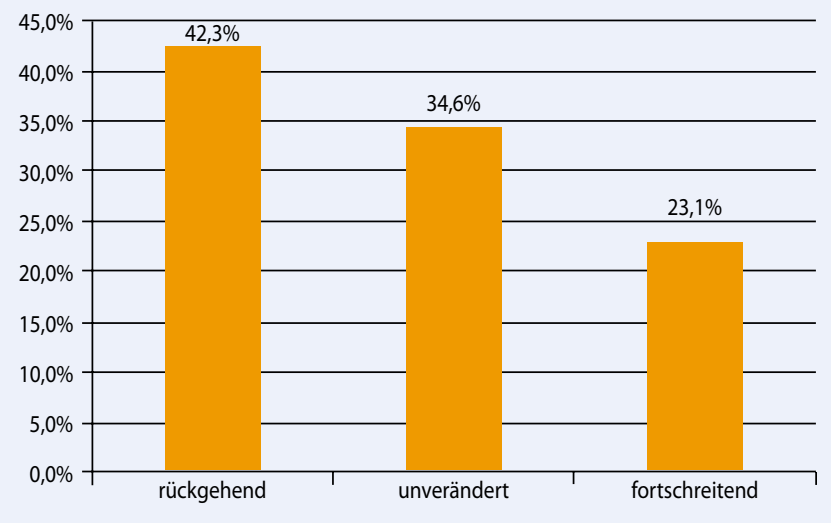

Abb. $4 \Delta$ Bewertung des Gesamteindrucks der Läsionen anhand standardisierter, kalibrierter Röntgenbilder im gepaarten, aber verblindeten, randomisierten Verfahren (Befund nach 6 Monaten im Vergleich zum Ausgangsbefund). Die Untersucher bewerteten 20 der 26 Läsionen $(76,9 \%)$ als unverändert oder rückgehend (remineralisierend)

gleich von verblindetem Ausgangs- und Endbefund beurteilt.

Als Bewertungskategorien für den Gesamteindruck standen die Begriffe unverändert (Punktezahl 0), rückgehend $(+1)$ oder fortschreitend ( -1$)$ zur Verfügung. Betrug die Summe aus den drei Einzelauswertungen +3 oder +2 , wurde die Läsion final der Kategorie rückgehend zugeordnet, betrug die Summe +1 oder 0 oder -1 , der Kategorie unverändert, betrug sie -2 oder -3, der Kategorie fortschreitend.

Beim sekundären Endpunkt (Flächenveränderung) ordneten die drei Untersucher die Läsionen im Konsensverfahren wie an der Baseline den Kategorien E0,
E1, E2 oder D1 zu [15]. Die Auswertung beider Endpunkte erfolgte nach Entblindung durch eine weitere Person.

\section{Ergebnisse}

Die Auswertung der Bissflügelaufnahmen 6 Monate nach der Behandlung ergab in Bezug auf die globale Veränderung der Läsionen (Betrachtung von Strahlendurchlässigkeit und Läsionsfläche, primärer Endpunkt) folgende Ergebnisse (• Abb.4): Insgesamt 11 der 26 Läsionen wurden gegenüber dem Anfangsbefund als rückgehend eingestuft $(42,3 \%)$, 9 als unverändert $(34,6 \%)$ und $6(23,1 \%)$ als fortschreitend. Die Übereinstimmung ( $\kappa$-Wert) zwischen den Untersuchern war mit 0,56 deutlich. Ein Beispiel für eine unveränderte Läsion zeigt $\bullet$ Abb. 6, für eine rückgehende Läsion • Abb. 7.

Wurde die Veränderung der Läsionsfläche (sekundärer Endpunkt) zwischen Ausgangs- und Endbefund auf Basis der Ausdehnung in den Kategorien E0, E1, E2 und D1 betrachtet, ergab sich ein Anteil von 17 unveränderten Läsionen $(65,4 \%)$, gegenüber 8 verkleinerten $(30,8 \%)$ und 1 vergrößerten Läsion (3,8\%; • Abb. 5a). Im Einzelnen blieben 3 von 5 E1-Läsionen (äußere Schmelzhälfte) und 14 von 20 E2-Läsionen (innere Schmelzhälfte) unverändert ( $\bullet$ Abb. 5b). Bei den E1-Läsionen wurde 1 vollständige Remission (keine kariös bedingte Strahlendurchlässigkeit mehr erkennbar, E0) und 1 Fortschreiten zur Kategorie E2 registriert. Bei 6 E2-Läsionen wurde eine Verkleinerung der Läsionsfläche beobachtet. Sie wurden nun als E1 (4) bzw. E0 (2) kategorisiert. Bei der D1-Läsion wurde dasselbe Phänomen beobachtet, sie wurde nach 6 Monaten als E2-Läsion eingestuft.

\section{Diskussion}

Die Zwischenergebnisse dieser prospektiven, unkontrollierten Studie deuten darauf hin, dass mithilfe der biomimetischen Mineralisation eine partielle oder sogar vollständige Remission kariöser Entkal- 


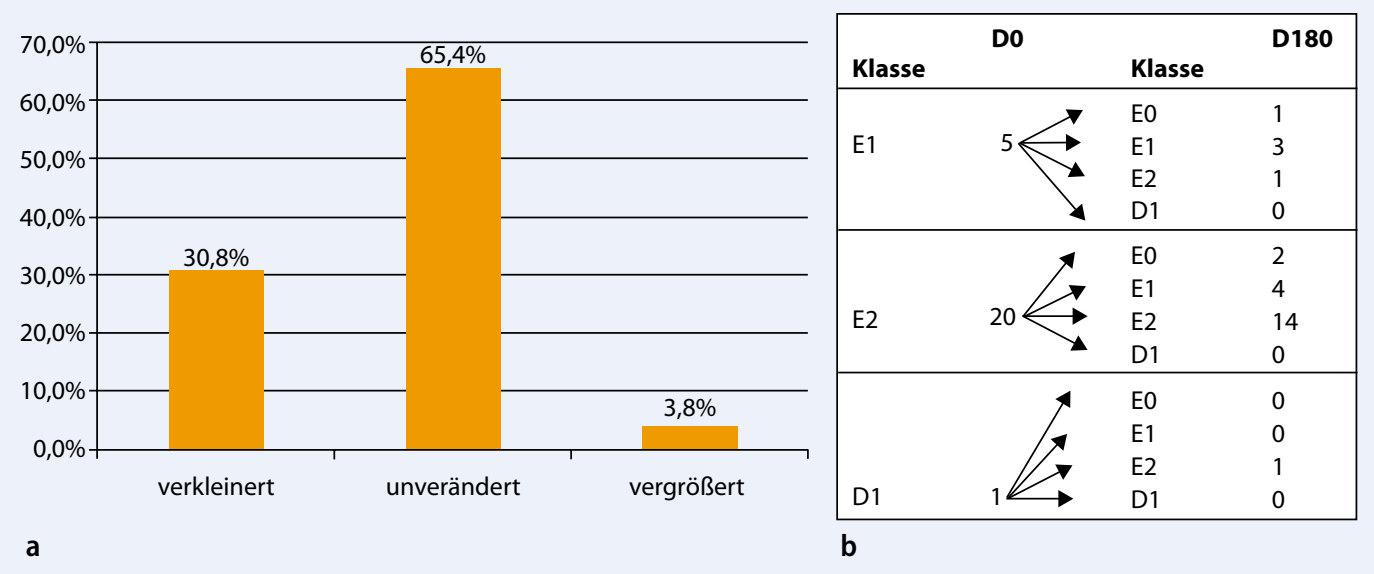

Abb. $5 \Delta$ Bewertung der Flächenveränderung der Karies nach 6 Monaten (D180) im Vergleich zum Ausgangsbefund (D0) anhand standardisierter, kalibrierter Röntgenbilder im gepaarten, aber verblindeten, randomisierten Verfahren. a Darstellung als Solldiagramm. b Entwicklung der Kariesläsionen in Bezug auf deren Klasse (E0, E1, E2, D1) vom Ausgangsbefund (D0) zum Befund nach 6 Monaten (D180). Hier gab es nur bei einer der 26 Flächen eine Vergrößerung der Läsionsfläche von der äußeren in die innere Schmelzhälfte (E1 $\rightarrow E 2,3,8 \%$ der Läsionen)
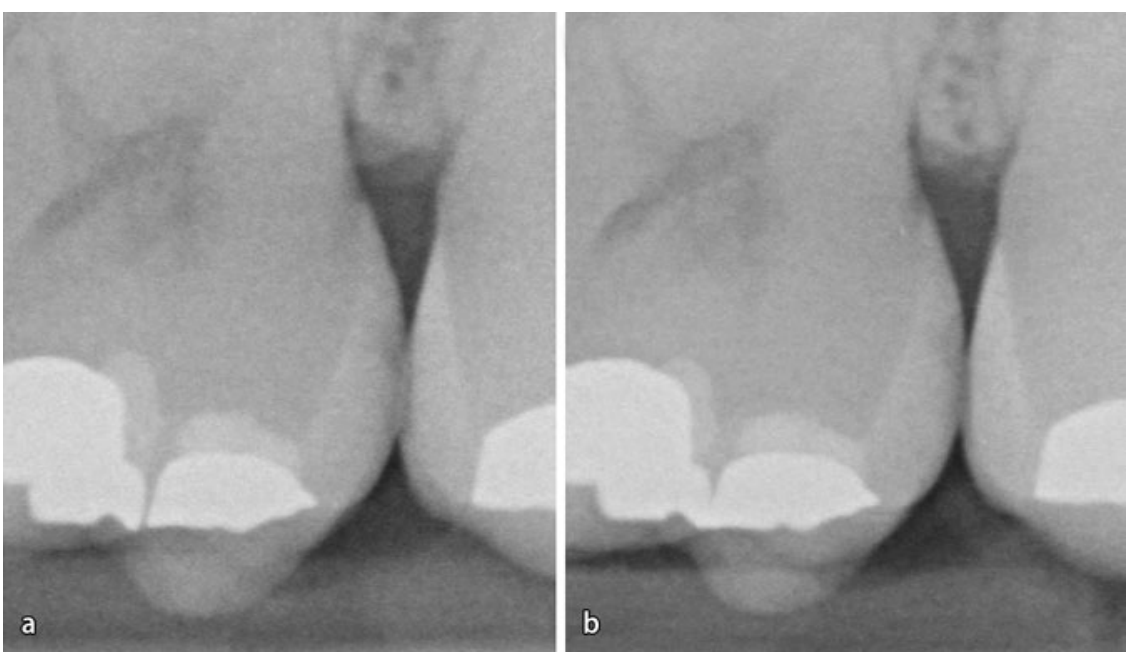

Abb. $6 \Delta$ Beispiel für eine radiologisch stabile Läsion (Zahn 26 distal). a Ausgangsbefund. b Keine sichtbare Veränderung der Fläche und Strahlendurchlässigkeit 6 Monate nach Behandlung
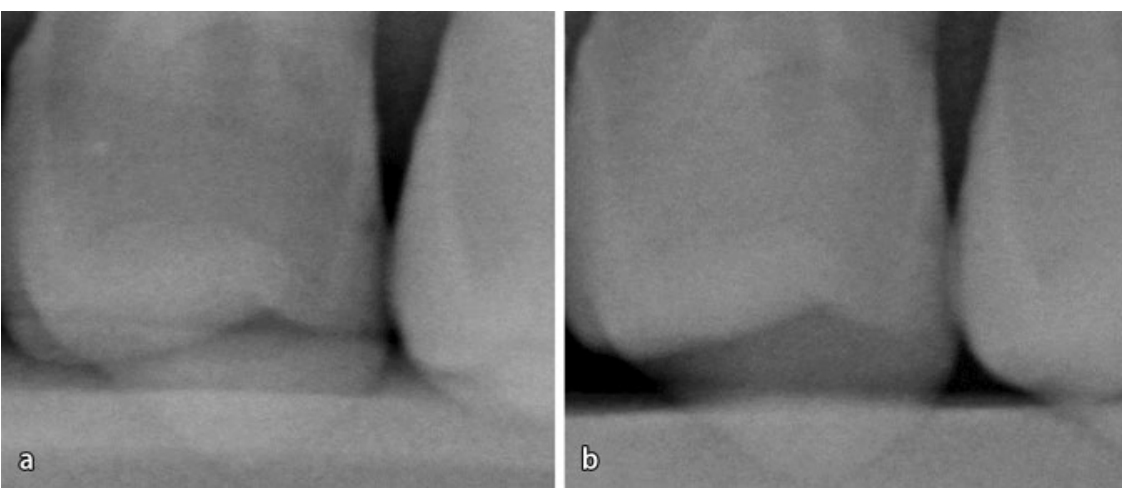

Abb. 7 \ Beispiel für eine Läsion, die als rückgehend (remineralisiert) eingeschätzt wurde (Zahn 24 distal). a Ausgangsbefund. b Sichtbare Verkleinerung der Fläche und zugleich Abnahme der Strahlendurchlässigkeit 6 Monate nach Behandlung kungen bis in die innere Schmelzhälfte gelingen kann. Dies gilt für initiale Läsionen im Schmelzbereich ohne Kavitation auf der Basis des Gesamteindrucks (primärer Endpunkt) und der Veränderung der radiopaken Fläche (sekundärer Endpunkt) in Bissflügel- oder Zahnfilmaufnahmen. Damit wurden die In-vitro-Ergebnisse der grundlegenden Publikation zu der neuen Methode in der Zeitschrift Journal of Dental Research [12] und einer weiteren, aktuellen Untersuchung [11] bestätigt. Beide zeigen ebenfalls eine Mineralisation in der Tiefe des Schmelzdefekts. Damit könnte die Forderung nach einer klinisch relevanten Remineralisation unterhalb der Läsionsoberfläche mit der vorgestellten Methode erstmals erfüllt werden [25].

\section{\) Die Resultate zeigen} eine Mineralisation in der Tiefe des Schmelzdefekts

Die Röntgenopazität einer Läsion korreliert mit dem Mineralgehalt und ist daher ein zuverlässiger Parameter, mit dem sich De- oder Remineralisation bestimmen lässt [13]. Insofern kann eine Verbesserung des Gesamteindrucks oder eine Flächenverkleinerung im Röntgenbild als Mineralisation interpretiert werden. Eine Remineralisation initialkariöser Läsionen erfolgt mit bisherigen präventiven Verfahren, oder wenn nur die im Speichel ent- 
haltenen Minerale einwirken, in den äuBeren $40 \mu \mathrm{m}$ und ist daher vermutlich radiologisch nicht nachweisbar [1]. Eine Mineralisation ist aufgrund publizierter Untersuchungen zur biomimetischen Mineralisation auch jenseits dieser Schichttiefe anzunehmen, was in der vorliegenden Untersuchung bestätigt wurde.

Die durchschnittliche Dicke approximaler, nichtkavitierter Schmelzläsionen beträgt etwa $350 \mu \mathrm{m}$ [20], sodass bei Verbesserung der Läsionsfläche von einer E2- zu einer E0-Läsion (2 Läsionen in der vorliegenden Studie) mit biomimetischer Mineralisation eine deutlich größere Tiefenwirkung angenommen werden kann als bei bisher verfügbaren Produkten. Für vergleichbare Verfahren wie approximale Versiegelung und Infiltration erfolgte die Auswertung ebenfalls mithilfe von Röntgenaufnahmen. Mögliche alternative Verfahren wie die digitale fiberoptische Transillumination (DiagnoCam, KaVo, Biberach, Deutschland) sind noch nicht ausreichend dokumentiert.

Nur eine der untersuchten Läsionen zeigte im Röntgenbild eine Verschlechterung, also eine Vergrößerung der radiopaken Fläche. Dabei handelte es sich um eine Erweiterung von der äußeren (E1) in Richtung innere Schmelzhälfte (E2, - Abb.5b). Beim Gesamteindruck der Veränderung bewerteten die drei Untersucher dagegen 23,1\% der Läsionen als fortschreitend im Vergleich zum Ausgangsbefund ( $\bullet$ Abb. 4). Der Parameter klinisch-radiologischer Gesamteindruck wurde vorab als primärer Endpunkt definiert und ist als Goldstandard bei der Einschätzung von Approximalkaries anzusehen. Daher muss in der vorliegenden Zwischenauswertung von einem Anteil rückgehender, also remineralisierender Läsionen von $42,3 \%$ ausgegangen werden.

Die evidente Abweichung zwischen Gesamteindruck und Flächenanalyse erklärt sich dadurch, dass beim primären Endpunkt neben der auf den Röntgenfilm projizierten Fläche Veränderungen bei der Strahlendurchlässigkeit der Läsion als Teil des Gesamteindrucks bewertet wurden. Die Strahlendurchlässigkeit wurde in der vorliegenden Zwischenauswertung nicht separat bestimmt. In der abschließenden Auswertung nach Ablauf von 12 Monaten soll dies jedoch mithil- fe der digitalen Subtraktionsradiographie nachgeholt werden.

\section{Vergleich mit anderen Methoden}

Hinsichtlich der Indikation Initialkaries lassen sich Fluoridierung, Versiegelung und Kunststoffinfiltration am ehesten mit der biomimetischen Mineralisation vergleichen. Fluoride hemmen in Abhängigkeit vom $\mathrm{pH}$-Wert die Entkalkung und fördern die Remineralisation mit im Speichel vorhandenem Kalzium und Phosphat an der Oberfläche [26]. Dieses Wirkprinzip von Fluoriden wird auch als anorganisch-chemisch bezeichnet [6]. Dagegen ist bei der biomimetischen Mineralisation mit den selbstorganisierenden Peptiden zusätzlich eine organische Komponente wirksam. Die Bildung der Biomatrix mit Einlagerung von Mineralen imitiert die Vorgänge bei der Schmelzentstehung und ähnelt daher einem regenerativen Vorgang [12]. Um den Zugang zum Läsionskörper zu ermöglichen, muss die Schmelzoberfläche mit Phosphorsäure vorbehandelt werden. Starke Säuren oder Kunststoffe kommen nicht zum Einsatz.

\section{I) Die Bildung der \\ Biomatrix ähnelt einem \\ regenerativen Vorgang}

Approximale Versiegelung und Kunststoffinfiltration sind an die chemisch-mechanische Adhäsivtechnik mit Kompositen angelehnt. Für beide Verfahren konnte im günstigen Fall eine Stabilisierung des Ausgangsbefunds gezeigt werden [15, 16, 19]. Das bedeutet, dass die verwendeten Adhäsive und Kunststoffe ein weiteres Fortschreiten der Karies verhinderten. Eine Remission im Sinne einer Wiederherstellung der Schmelzstruktur ist schon methodisch bedingt nicht möglich. Andererseits war bei beiden Methoden der Anteil fortschreitender Läsionen im Vergleich zur biomimetischen Mineralisation geringer $[16,19]$.

Die klinische Sicherheit der biomimetischen Mineralisation wurde bereits in einer klinischen Studie dokumentiert [2]. Dabei wurden zwei Fälle von Dentinüberempfindlichkeit registriert, die möglicherweise auf die Reinigungsschritte zu- rückzuführen sind. In der vorliegenden Studie waren keine Nebenwirkungen und keine vorübergehende Dentinüberempfindlichkeit festzustellen.

\section{Fazit}

\section{- Mit der biomimetischen Minerali- sation steht erstmals ein Verfahren zur Verfügung, mit dem eine Wie- derherstellung verloren gegangener Schmelzkristalle unter der Oberflä- che, d. h. im Läsionskörper angestrebt wird. \\ - Die vorliegende Untersuchung an ap- proximalen Initialläsionen deutet da- rauf hin, dass diese in vitro gesicherte Methode auch klinisch relevante Er- gebnisse erzielen kann. \\ - Längerfristig angelegte, kontrollier- te Studien mit entsprechenden Ver- gleichsgruppen stehen noch aus.}

\section{Korrespondenzadresse}

\section{Dr. M. Schlee}

Praxis Dr. Markus Schlee

Bayreuther Str. 39, 91301 Forchheim

Deutschland

m.schlee@32schoenezaehne.de

\section{Einhaltung ethischer Richtlinien}

Interessenkonflikt. M. Schlee hat wirtschaftliches Interesse am Produkt, J.H. Koch ist freier Fachautor und Berater von credentis ag, C. Bommer ist Angestellte von credentis ag; F. Rathe, T. Huck, T. Schad und A. Tjaden geben an, dass kein Interessenkonflikt besteht; die Studie wurde von credentis ag unterstützt. Alle in diesem Beitrag beschriebenen Untersuchungen am Menschen wurden mit Zustimmung der zuständigen EthikKommission, im Einklang mit nationalem Recht sowie gemäß der Deklaration von Helsinki von 1975 (in der aktuellen, überarbeiteten Fassung) durchgeführt. Von allen beteiligten Patienten liegt eine Einverständniserklärung vor.

\section{Open Access}

This article is distributed under the terms of the Creative Commons Attribution License which permits any use, distribution, and reproduction in any medium, provided the original authors and the source are credited.

\section{Literatur}

\footnotetext{
1. Bergman G, Lind PO (1966) A quantitative microradiographic study of incipient enamel caries. J Dent Res 45:1477-1484
} 
2. Brunton PA, Davies RP, Burke JL et al (2013) Treatment of early caries lesions using biomimetic selfassembling peptides - a clinical safety trial. $\mathrm{Br}$ Dent J 215:E6

3. Cochrane NJ, Cai F, Huq NL et al (2010) New approaches to enhanced remineralization of tooth enamel. J Dent Res 89:1187-1197

4. Do Valle D, Ekstrand KR (1999) The fate of approximal lesions in young adults during an 8-12-year period: a radiographic examination. Caries Res 33:228

5. Eakle WS, Featherstone JD, Weintraub JA et al (2004) Salivary fluoride levels following application of fluoride varnish or fluoride rinse. Community Dent Oral Epidemiol 32:462-469

6. Ellwood R, Fejerskov O, Cury JA et al (2008) Fluorides in caries control. In: Fejerskov 0 , Kidd EA (eds) Dental caries. The disease and its clinical management. Blackwell Munksgaard, Oxford, pp 287-327

7. Featherstone JD (2009) Remineralization, the natural caries repair process - the need for new approaches. Adv Dent Res 21:4-7

8. Featherstone JD, White JM, Hoover $\mathrm{Cl}$ et al (2012) A randomized clinical trial of anticaries therapies targeted according to risk assessment (caries management by risk assessment). Caries Res 46:118129

9. Griffin SO, Regnier E, Griffin PM et al (2007) Effectiveness of fluoride in preventing caries in adults. J Dent Res 86:410-415

10. Hahn P (2011) Aktuelle Entwicklungen in der Kariesprophylaxe. Freie Zahnarzt 54-62

11. Kind L, Wuethrich A, Stevanovic S et al (2013) A self-assembling peptide with the potential of noninvasive regeneration of early caries lesion. Poster abstracts of the 6th biennial meeting of the European Federation of Conservative Dentistry (EFCD) held in association with the 33th national congress of the College National des Enseignants en Odontologie Conservatrice (CNEOC), France, 9-11 May 2013, Paris, France. Clin Oral Investig 17

12. Kirkham J, Firth A, Vernals D et al (2007) Self-assembling peptide scaffolds promote enamel remineralization. J Dent Res 86:426-430

13. Klinger HG, Wiedemann W (1985) A method for radiographic longitudinal study of mineral content during in-vitro demineralization and remineralization of human tooth enamel. Arch Oral Biol 30:373-375

14. Lussi A, Hellwig E, Klimek J (2012) Fluoride - Wirkungsmechanismen und Empfehlungen für deren Gebrauch. Schweiz Monatsschr Zahnmed 122:1037-1042

15. Martignon S, Ekstrand KR, Ellwood R (2006) Efficacy of sealing proximal early active lesions: an 18-month clinical study evaluated by conventional and subtraction radiography. Caries Res 40:382388

16. Martignon S, Ekstrand KR, Gomez J et al (2012) Infiltrating/sealing proximal caries lesions: a 3-year randomized clinical trial. J Dent Res 91:288-292

17. Mejare I, Stenlund H, Zelezny-Holmlund C (2004) Caries incidence and lesion progression from adolescence to young adulthood: a prospective 15-year cohort study in Sweden. Caries Res 38:130-141

18. Meyer-Lückel H, Ekstrand K, Paris S (2012) Karies: Wissenschaft und klinische Praxis. Thieme, Stuttgart

19. Meyer-Lueckel H, Bitter K, Paris S (2012) Randomized controlled clinical trial on proximal caries infiltration: three-year follow-up. Caries Res 46:544548
20. Meyer-Lueckel H, Paris S, Kielbassa AM (2007) Surface layer erosion of natural caries lesions with phosphoric and hydrochloric acid gels in preparation for resin infiltration. Caries Res 41:223-230

21. Micheelis W, Schiffner U, Hoffmann T et al (2006) DMS IV: Zusammenfassung/Abstract. In: IDZ IdDZ (Hrsg) Vierte Deutsche Mundgesundheitsstudie (DMS IV). Materialienreihe, Bd 31. Deutscher Ärzteverlag, Köln, S 17-21

22. Nyvad B, Machiulskiene V, Baelum V (2003) Construct and predictive validity of clinical caries diagnostic criteria assessing lesion activity. J Dent Res 82:117-122

23. Petersen PE (2003) The World Oral Health Report 2003: continuous improvement of oral health in the 21st century - the approach of the WHO Global Oral Health Programme. Community Dent Oral Epidemiol 31(Suppl 1):3-23

24. Pitts NB (2004) Are we ready to move from operative to non-operative/preventive treatment of dental caries in clinical practice? Caries Res 38(3):294304

25. Pitts NB, Wefel JS (2009) Remineralization/desensitization: what is known? What is the future? Adv Dent Res 21:83-86

26. Ten Cate JM, Duijsters PP (1983) Influence of fluoride in solution on tooth demineralization. I. Chemical data. Caries Res 17:193-199

27. Van Nieuwenhuysen JP, D'hoore W, Carvalho J et al (2003) Long-term evaluation of extensive restorations in permanent teeth. J Dent 31:395-405

28. Yengopal V, Mickenautsch S (2009) Caries preventive effect of casein phosphopeptide-amorphous calcium phosphate (CPP-ACP): a meta-analysis. Acta Odontol Scand 67:321-332 